\title{
Lightweight Signaling and Efficient Coupling Heuristic for Optical Star Networks MAC Protocols
}

\author{
Maurice GAGNAIRE \\ Ecole Nationale Supérieure des Télécommunications \\ 46 rue Barrault - 75634 Paris cedex 13 - FRANCE \\ tel: (33) (1) 45.81.74.11 - Fax: (33) (1) 45.89.16.64 - Email : gagnaire@res.enst.fr \\ February 24-25, 1997
}

\section{IFIP Working-Conference on Optical Network Design and Modelling Vienna-Austria}

\begin{abstract}
Several MAC protocols have been proposed to prevent collisions on passive optical star networks. Time Division Multiple Access (TDMA) is a simple way to guarantee conflict-free concurrent transmissions on such networks. Because it does not require any signaling, TDM efficiency is not distance dependant. However, TDM suffers from non negligible access delays under low load. To reduce this drawback, dynamic allocation schemes (DAS) such as the Random Sheduling Algorithm (RSA) or the Group Time Division Multiplexing (GTDM) have been proposed. Both of these mechanisms reserves slots on a packet-bypacket basis by means of a signaling channel. The capacity in bit/s of this signaling channel is directly related to the number of buffers used in each node. The parallel queueing strategy allows to reduce the signaling cost by using a small number of buffers in each node. In that case, coupling algorithms such as the Minimum-degree Vertex First Scheduling (MVFS) have been proposed to manage the packets enqueueing/dequeueing process. In this paper, we propose a new MAC protocol for passive optical star networks which cumulates the benefits of GTDM in terms of lightweight signaling with those of the MVFS algorithm in terms of coupling efficiency. The performance of this new protocol is compared to other existing solutions by means of computer simulations. We also underline the disruptive influence of propagation delays on the DAS-type protocols efficiency.
\end{abstract}

Keywords : Single-hop networks, passive optical star, MAC protocols, performance evaluation. 


\section{Introduction}

Broadband interactive applications require new network generations for both local area networks (LAN) and long distance networks (W.ANs). The challenge to satisfy is double : how to offer to each session between such applications a very large bandwidth while maintaining simultaneously a very short network response time. New LANis like Fast Ethernet, 100-VG Any-LAN or FDDI are a first but a limited step in that. direction. Three new approaches partially answer this challenge in the field of local area networks. In the the short term, Gigabit-LANs and ATM-LANs look like possible solutions. Like classical LANs, Gigabit-LANs are based on a broadcasting medium. Metaring [1], ATM-Ring [3] or CRMA-2 [2] are some examples of Gigabit-LANs. These networks allow a significant increase in the medium capacity up to the Gigabit range by means of slot reuse, concurrent transmisions and multiple path routing. Unlike Gigabit-LANs of which capacity is given by the medium bandwidth, ATM-LANs offer a better scalability. Indeed, a load increase on an ATM-LAN may be satisfied by adding new switches and new links. Nevertheless, Gigabit-LANs and ATM-LANs are limited in terms of bandwidth and of response time because of the electro-optic bottleneck. For both of them, the speed of electronics must be of the same order of magnitude as the aggregate throughput on each link. Wavelength Division Multiplexing (WDM) is a very promising alternative to overtake this limitation in bandwidth. Instead of using a single channel per link with a high bit rate, WDM allows to create several optical channels of a few Gigabit/s on a same fiber. A specific wavelength is associated to each of these channels between a sender and a receiver. The speed of electronics in an endstation is then given by the speed of a single optical channel. In high speed networks, propagation delay is predominant over transmission delay ${ }^{1}$. For a given optical link between two nodes, network reponse time is given by the summation of the cumulated propagation delay $T_{p}$ and of the cumulated nodes latency $T_{l}$. If it is not possible to improve $T_{p}$, a reduction in $T_{l}$ may be obtained by replacing active connections by passive ones. Passive optical connections guarantee a null station latency. On the other hand, fast tunable transmitters/receivers facilitate a dynamic allocation of wavelengths among the various couples sender/receiver. Single-hop all-optical LANs turn to good account of all these improvements.

Several topologies have been investigated for single-hop all-optical LANs. In this paper, one considers the case of passive optical star coupler networks. One assumes that stations use fixed transmitters and tunable receivers. Contentions may occur if two users want to communicate simultaneously with a same destination. Different medium access protocols (MAC) have been proposed for conflict-free access to passive optical star networks. Most of them use a synchronization between nodes allowing a slotted access. Preassigned wavelentgth allocation based on a Time Division Multiple Access scheme is the simplest way to prevent any contention on passive optical star networks. Such a MAC protocol does not optimize access delays in case of low loads. To reduce this drawback, Dymamic Allocation Schemes (DAS) have been proposed in the litterature. In each station $i$, generated packets are stored in distinct buffers according

\footnotetext{
${ }^{1}$ This duality is called delay $\times$ bandwidth product.
} 
to their destination address. The state of the network at each instant is given by the amount of packets in these buffers. Dynamic allocation schemes assume that at the beginning of each slot, each station has a partial knowledge of the state of these queues. A signaling channel must be used for that purpose. In section 2 , we recall the main characteristics of passive optical star networks. We describe the principle and underline the limits of the TDM access. In section 3, two dynamic allocation schemes (DAS) are presented : the Random Sheduling Algorithm (RSA) and the Group Time Division Multiplexing (GTDM). Both of these schemes reserve slots on a packet-by-packet basis. The cost in bits of the signaling channel for such dynamic schemes strongly limits the amount of nodes that may be connected to the network. New buffering strategies have been investigated to solve this problem. The Parallel Queueing technique which is one of them is presented in section 4. Some algorithms such as the Minimum-degree Vertex First Scheduling (MVFS) must be associated to parallel queueing for managing the enqueueing and the dequeueing of the various buffers in each node. In section 5 , we show that the MVFS allows a much better coupling efficiency between nodes than the R.S. We then propose a new MAC protocol for passive optical star networks which cumulates the advantages of GTDM in terms of signaling cost and of the MVFS in terms of coupling efficiency. Several simulation results are presented and commented to compare the performance of the GTDM+MVFS scheme with other existing protocols. Finally, we conclude this paper in section 6 by underlining the disruptive influence of propagation delays on the performance of DAS-type protocols.

\section{Passive optical star networks with TDM access}

\subsection{Configuration}

In single-hop all-optical networks, a source node is connected with a destination node by means of an end-to-end optical channel. Passive folded bus and passive optical star are two possible topologies for single-hop all-optical networks. Both of these topologies uses $2 \times 1$ and $1 \times 2$ passive optical couplers. Figure 1 illustrates a passive optical star configuration with $N=4$ stations.

Unlike folded bus topology, optical-star topology allows a fixed and low attenuation between any couple source/destination. Each wavelength $\lambda_{i}$ associated to a transmitter $i$ is broadcast toward all the stations of the network. Let $P$ be the optical power of a transmitter. Let $\alpha$ and $\beta$ be respectively the attenuation of $2 \times 1$ and $1 \times 2$ couplers. Any receiver detects optical signals with a power $\alpha^{2} . P . \beta^{2}$. Let $j$ be the index of the destination node of a packet sent by node $i$. By means of a tunable receiver, node $j$ is able to select the wavelength $\lambda i$ among the composite spectrum it receives. One assumes here that the receiver is informed by the transmitter of the tuning wavelength. Either this information is given before each packet transmission (optical packet switching), or before the opening of a communication (optical circuit switching). Because receivers can only tume to a single wavelength at a given instant, contention may occur if several stations try to transmit simultaneously to the same receiver. 


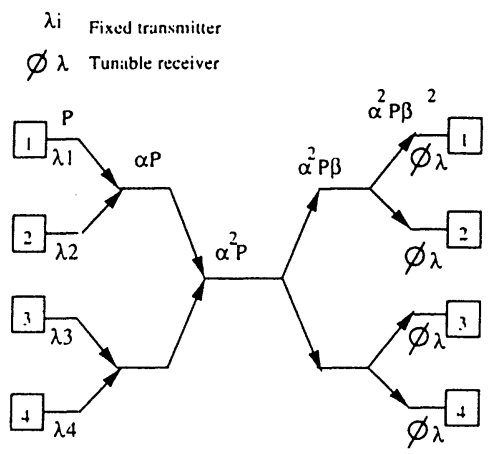

Figure 1: Principle of the passive optical star coupler network.

\subsection{The Preassigned Time Division Multiplexing (TDM)}

Time division multiplexing is the simplest way to prevent any collision on a passive optical star. The $N$ stations of the network are synchronized. Time is slotted in fixed intervals which impose a common packet size. For sake of simplicity, we assume that a station may send packets to itself. The packets generated by each node $i$ are stored in $N$ separated queues according to their destination address. Let $\Delta$ be the slot duration. A frame structure composed of $N$ slots is simultaneously broadcasted to the each station. Figure 2 illustrates the principle of preassigned TDM access. At instant $t_{1}$, which is the beginning of the first slot of a frame, stations $1,2, \ldots N$ are respectively allowed to transmit a packet to stations $1, N, N-1, \ldots 2$. Using a cicular permutation in the sucessive slots assignment, one may notice that each station is allowed to send at most one packet per frame to any receiver. TDM access is well suited for optical circuit switching. Because it does not require any signaling, TDM access is insensitive to distance. The price of this simplicity is paid by non negligible access delays under low loads.

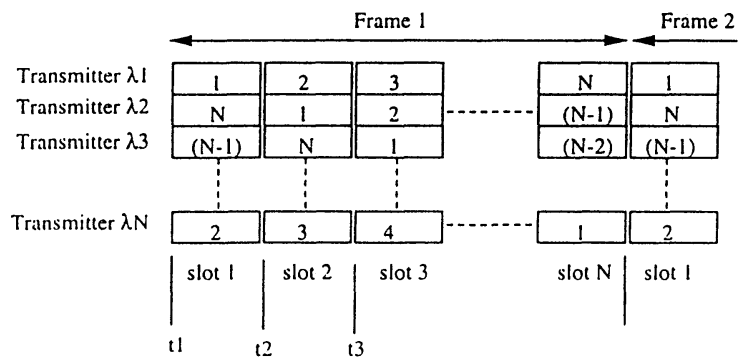

Figure 2: Principle of the TDM access on a passive optical star network. 


\section{Dynamic Allocation Schemes (DAS)}

Let us assume that time is slotted and that stations are synchronized. Optical packet switching requires that wavelength allocation is made on a packet-by-packet basis. In that case. a signaling channel is mandatory to inform during each slot $k$; on which wavelength each receiver will have to tune a few slots later. To prevent any collision, each node of the network must have, slot by slot. a partial knowledge of the state of the buffers of the $N-1$ other nodes. On the basis of this knowledge, all the stations run a common algorithm to decide on which received wavelength they have to tune. Two main problems appear in that case. First, the amount of necessary bits to broadcast slot by slot the information relative to the state of the buffers is limited because of the finite capacity of the signaling channel. Secondly, the shorter the slot duration, the more influent the propagation delays of signaling information on data packet access delays. Let us consider for instance a packet size equivalent to the payload of an ATM cell ( 48 bytes). If each transmitter allows a throughput of $1 \mathrm{Gbit} / \mathrm{s}$, a slot duration is then 384 ns. Assuming a signal velocity $\mathcal{V}$ on the optical fibre of $180.000 \mathrm{Km} / \mathrm{s}$, distance between a sender and a receiver must be less than 70 meters. Thus, knowing the state of the buffers at the beginning of slot $k$, stations are authorized to transmit at the beginning of slot $k+1$ only in the case of very short range networks. One assumes in the following the use of an out-of-band signaling. In addition to its fixed transmitter on wavelength $\lambda_{i}$, each station $i$ owns another fixed transmitter on a common wavelength $\lambda_{s}$. Likewise, in addition to its tunable receiver, each node $i$ owns a fixed receiver on the wavelength $\lambda_{s}$. Concurrent transmissions on this common signaling channel are made on the basis of a TDM access.

\subsection{The Random Scheduling Algorithm (RSA)}

The Random Scheduling Algorithm (RSA) has been proposed in [4] for wavelength allocation in dynamic allocation schemes. The RSA is based on a same random number generator seed implemented in each station of the network. As soon as they have received the information about the state of the buffers, all the stations run the RSA and mutually arrive to the same conclusion. Effective transmissions may occur at the best when the most distant sender and receiver have in their turn arrived to this same conclusion. The RSA is run at the beginning of every slot. Figure 3 gives an example of the buffers state between two successive slots in the case of a network with $N=3$ stations. Let $i$ be the index of the transmitters and $j$ be the index of the queue containing packets for node $j$. We assume that the slot duration on the signaling channel is identical and synchronized with the slot duration $\Delta$ on the data channels.

Figure 4 describes the principle of the out-of-band signaling channel associated to the RSA. We see on this figure the information sent on the signaling wavelength $\lambda_{s}$ by the $N$ stations while they are in the state given by Figure 3. A slot duration on the signaling channel is devided in $N$ minislots. Each minislot is itself devided in $N$ bits. By convention, the $i$-th minislot of each frame is dedicated to station $i$. The $j$-th bit in the $i$-th minislot is set to " 1 " if station $i$ has at least one packet waiting for 


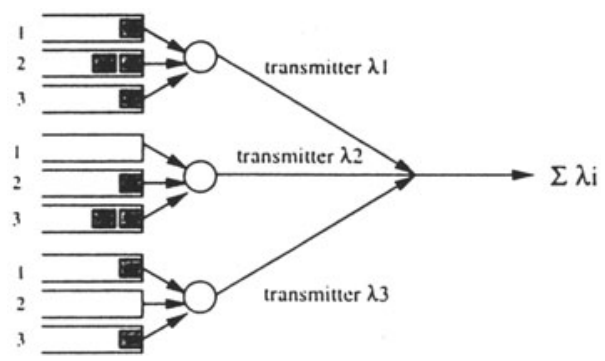

Figure 3: A possible state for the different queues of a network with 3 nodes.

transmission in its queue $j$. If it is not the case, this bit remains to " 0 ".

We see from Figure 4 the influence of signaling propagation delays on data packet access delays. Let $\Omega$ be the set $\{1,2, \ldots, N\}$. The Random Scheduling Algorithm is then :

1. A transmitter $i_{1}$ is randomly selected in $\Omega$.

2. A queue $j_{1}$ is randomly selected among the nonempty queues of station $i_{1}\left(j_{1} \in\right.$ $\Omega$ ). At the end of the RSA execution, station $i_{1}$ will be allowed to send a data packet towards station $j_{1}$.

3. A second transmitter $i_{2}$ with $i_{2} \neq i_{1}$ is randomly selected among $\Omega$.

4. A queue $j_{2}$ with $j_{2} \neq j_{1}$ is randomly selected among the nonempty queues of station $i_{2}\left(j_{2} \in \Omega\right)$. At the end of the RSA execution, station $i_{2}$ will be allowed to send a data packet towards station $j_{2}$.

5. Steps 3 and 4 are repeated, each time by removing from $\Omega \times \Omega$ a new couple $\left(i_{x}, j_{x}\right)$, until all the $N$ stations have been considered.

Figure 5 illustrates by means of a bipartite graph the state of the queues corresponding to Figure 3. The left part and the right part of the graph respectively corresponds to the transmitting side and to the receiving side. For each node $i$, vertex $E_{i}$ corresponds to the fixed transmitter and vertex $R_{i}$ corresponds to the tunable receiver. An edge exists from vertex $E_{i}$ to vertex $R_{j}$ if there is at least one packet in station $i$ waiting to be transmitted towards station $j$.

Let us consider for instance a possible sequence $\alpha$ given by the RSA. This sequence $\alpha$ is illustrated by the bipartite graph given in Figure 6 :

$$
\alpha=\left\{\left(i_{1}=1, j_{1}=2\right),\left(i_{2}=3, j_{2}=1\right),\left(i_{3}=2, j_{3}=3\right)\right\}
$$

The RSA presents two major drawbacks. The cost in bit of the signaling information increases in $N^{2}$. These $N^{2}$ bits must be sent during a slot duration $\Delta$. If the signaling channel and the data channels have the same capacity in bit/s and if $L$ stands for the 


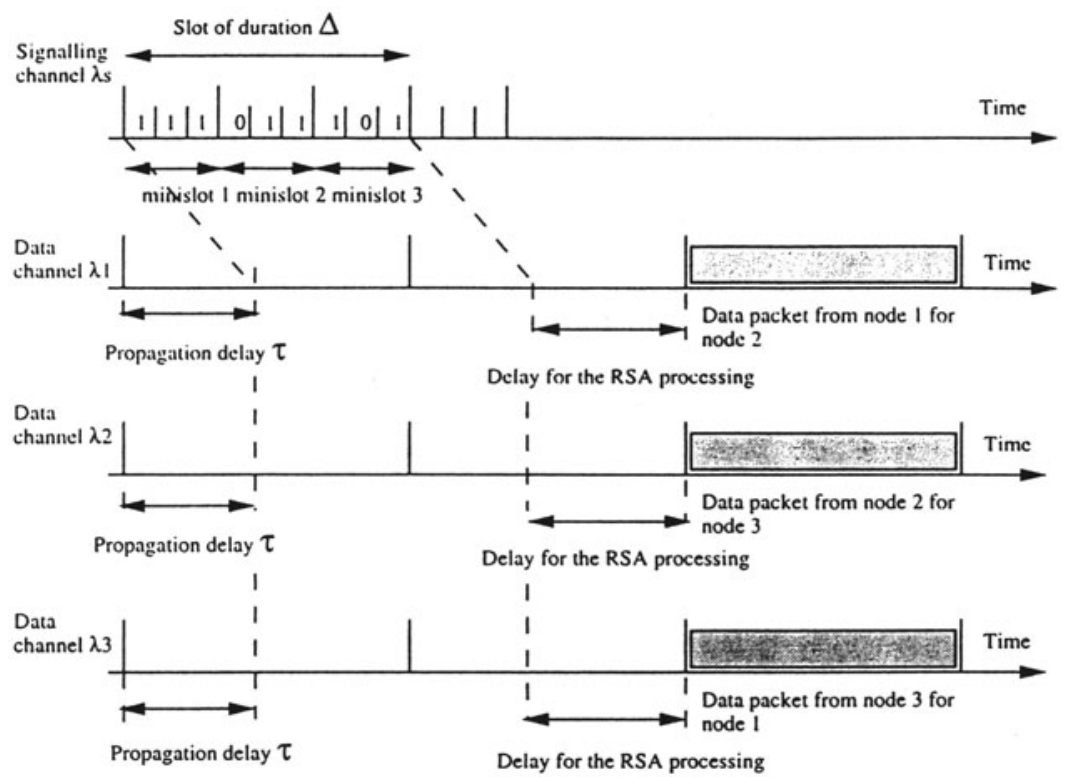

Figure 4: Time structure of the signaling channel and of the data channels of the RSA.

packet size in bits, the maximum number $N_{\max }$ of nodes that may be connected to the network is :

$$
N_{\max }=\lfloor\sqrt{L}\rfloor
$$

If $L=48$ bytes, then only up to 19 stations may be connected to the network. The non-optimal coupling between transmitters and receivers is the second drawback of the RSA. Figure 7 gives the bipartite graph associated to another possible random sequence $\alpha^{\prime}$ :

$$
\alpha^{\prime}=\left\{\left(i_{1}=1, j_{1}=2\right),\left(i_{2}=3, j_{2}=3\right),\left(i_{3}=2, j_{3}=?\right)\right\}
$$

The sequence $\alpha^{\prime}$ induces a waste in bandwitdth utilization because station 2 is not allowed to transmit.

\subsection{The Group Time Division Multiplexing (GTDM)}

Several algorithms have been proposed in the litterature so as to offer a better coupling efficiency than the RSA. The Hybrid Time Division Multiplexing (HTDM) is a combination of TDM and RSA [4]. The HTDM uses a frame divided in $N+M$ slots, where $N$ still stands for the number of nodes on the network. Globally, stations access the 


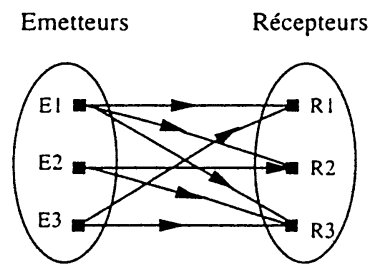

Figure 5: Bipartite graph associated to state of the different queues.

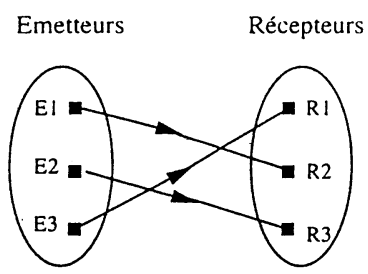

Figure 6: Bipartite graph associated to the random sequence $\alpha$.

networks by means of preassigned slots ( $N$ preassigned slots per frame). After every $N / M$ slots (with $N \geq M$ and $M$ is an integer), an "open" slot authorizes a station to transmit a packet to any receiver. To prevent contentions, open slots are allocated by means of the RSA. The HTDM reduces the cost of the signaling channel in comparison to the pure RSA. Indeed, the $N^{2}$ bits necessary to describe the state of the $N^{2}$ queues are transmitted during $N / M$ slots instead of one slot for the pure RSA. For a packet size $L$, the signaling cost limits the number $N_{\max }$ of stations that may be connected to the network such as :

$$
N_{\max }=\left\lfloor\frac{L}{M}\right\rfloor
$$

If $L=48$ bytes, several values of the couple $\left(N_{\max }, M\right)$ are possible : $(192,2),(96$, 4), $(48,8),(32,12)$.

The Group Time Division Multiplexing (GTDM) is also based on a combination of TDM and RSA [6]. The set of $N$ nodes is divided in several groups $g_{m}$ with $m \in$ $\{1,2, \ldots, K\}$ and $K \leq N$. Stations of group $g_{m}$ may communicate with stations of group $g_{n}$ during preassigned periods of time. Figure 8 illustrates the principle of GTDM. Again, stations are synchronized and time is slotted. A frame is devided into $K$ slots. At instant $t_{1}$, transmitters of group $g_{1}$ can send packets only to receivers of the same group, transmitters of group $g_{2}$ can send packets only to receivers of group $g_{K}$ and so on. A circular permutation in the successive slots assignment allows to prevent from any optical contention between the different groups. During each preassigned slot, the RSA is applied inside each group to determine the transmitter/receiver pairs for the 


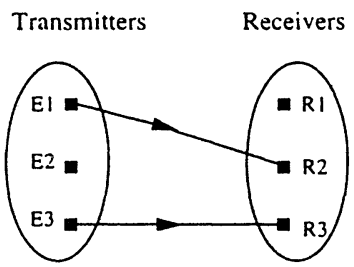

Figure 7: Bipartite graph associated to the random sequence $\alpha^{\prime}$.

next, slot. The signaling cost of GTDM is sensibly reduced compared to RSA. Instead of sending $N$ bits per slot, as with RSA, a station only sends $N / K$ bits per slot with GTDM. If $L$ is the data packet size, the signaling cost limits the number of stations that may be connected to the network to $N_{\max }$ such as :

$$
N_{\max }=\lfloor\sqrt{K \cdot L}\rfloor
$$

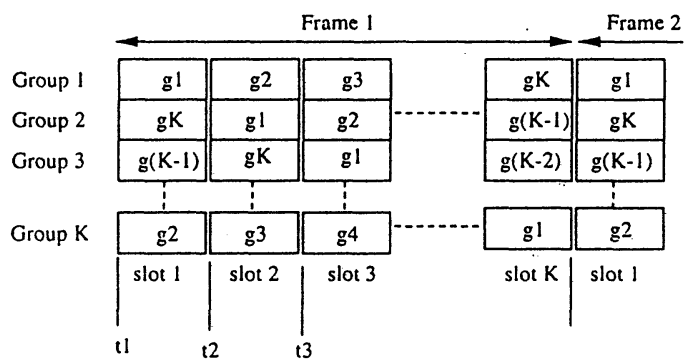

Figure 8: Principle of the Group TDM access on a passive optical star network

If $L=48$ bytes and $K=4, N_{\max }=38$ which is twice the capacity given by pure $\mathrm{RSA}$. In the case of symmetrical traffic, the groups are of the same size $N / K$ (if $N$ is even). In the case of asymmetrical traffic, the choice of the groups aims to minimize the contention probability. Thus, two stations generating many packets for a same destination should be placed in distinct groups.

\section{The Parallel Queue Scheduling}

The access protocols decribed above assume that in each node, generated packets are stored in different queues according to their destination address. The Parallel Queue scheduling proposed in [5] allows to use a reduced number of queues in each node by means of a two stage buffering. Like GTDM and RSA, parallel queue scheduling assumes a synchronization of the stations and a slotted access. An out-of-band signaling 
channel is also shared by the $N$ stations by means of a TDM access. This signaling channel uses a specific wavelength $\lambda_{s}$. Figure 9 describes the configuration of a station with Parallel Queue scheduling.

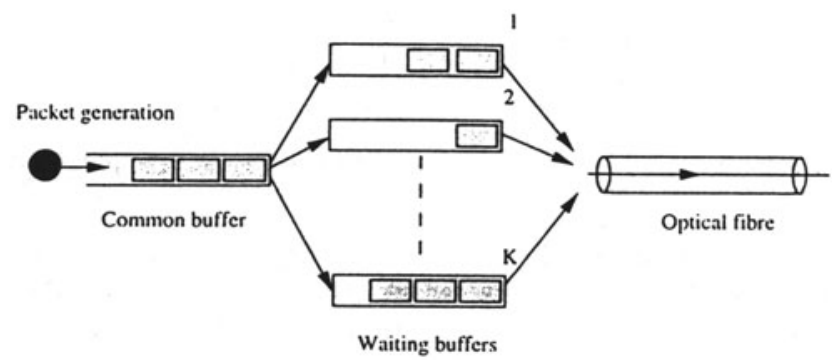

Figure 9: Principle of the Parallel Queue Scheduling.

The first stage is made of a large size FIFO buffer. The second stage is divided in $K$ parallel buffers (waiting buffers) with a same capacity $S$. The value of $K$ is fixed and low (5 or 6 ) for any network configuration. In each station $i$, generated packets are first of all stored in a the common FIFO buffer. At the beginning of each slot, the packet located at the head of the common buffer is enqueued in one of the waiting buffers. Two policies are proposed for the choice of the parallel buffer :

- Policy 1: The set of the $N$ receivers is divided into $K$ groups. A parallel queue is thus associated to a given set of destination adresses.

- Policy 2: The least loaded parallel queue is systematically chosen.

For both policies, a station broadcasts on the signaling channel the destination address of a packet as soon as this one has been transfered from the common buffer to one of the parallel queues. If policy 2 is adopted, a station also broadcasts the index of the chosen parallel queue. Thus, any station of the network knows slot by slot the state of the $K$ parallel queues of the $N-1$ other stations. This knowledge is then used to determine the transmitter/receiver pairs. Let $\Delta$ be the slot duration and $\tau$ be the propagation delay. A station is not allowed to send a packet before the corresponding signaling message has arrived to all the receivers. In other terms, this packet have to be buffered in a parallel queue at least during a delay $\tau$. The minimum size $S$ of the parallel queues is then given by :

$$
S=\left\lceil\frac{\tau}{\Delta}\right\rceil
$$

The cost of the signaling channel is given by the number of bits to be sent during each slot duration. In the case of policy 1 , one assumes that an address "0" corresponds to an empty common buffer. The signaling cost is then $N \cdot \log _{2}(N+1)$. In the case of policy $2, \log _{2} K^{\prime}$ bits are necessary to identify in a given node the used parallel queue. 
The signaling cost is then $N \cdot \log _{2}(N+1)+N \cdot \log _{2} K$. If $L$ is the data packet size, the signaling cost limits the number $N_{\text {max }}$ of stations that may be connected to the network. In the case of policy 1 , one has :

$$
N_{\text {max }} \cdot \log _{2}\left(N_{\text {ma: }}+1\right) \leq L
$$
by:

If $L=48$ bytes, then $N_{\max }=63$. In the case of policy 2 , the value of $N_{\max }$ is given

$$
N_{\max } \cdot \log _{2} K^{\circ} \cdot\left(N_{\max }+1\right) \leq L
$$

After all the stations have been informed of the presence or of the absence of a packet in the parallel queues, a simple heuristic algorithm given in [5] determines the transmitter/receivers pairs. This heuristic called the Minimum-degree Vertex First Scheduling (MVFS) is described below. Let us consider the bipartite graph including the set $T$ of the transmitters (left part of the graph with vertices $E_{i}$ ) and of the set $R$ of the receivers (right part of the graph with vertices $R_{j}$ ). At most $K$ edges link a vertex $E_{i}$ to $K$ vertices $R_{j}$. The different steps of the MVFS are the following ones:

1. Sort the vertices of $R$ according to the increasing order of the number of edges arriving on each of them.

2. Select randomly one vertex $R_{j 1}$ among those with the lowest order.

3. Select randomly one vertex $E_{i 1}$ among those linked to $R_{j 1}$ with an edge.

4. Remove $E_{i 1}, R_{j 1}$ and all the edges originated from $E_{i 1}$ and all the edges arriving at $R_{j 1}$.

5. Repeat steps 1 to 4 until all the stations have been consulted.

\section{The GTDM+MVFS scheme : a new access pro- tocol}

\subsection{The MVFS coupling efficiency}

We propose to associate the MVFS heuristic with the GTDM so as to cumulate the benefits of each of these mechanisms. Compared to pure RSA, GTDM allows a lightweight signaling. The higher the number of groups, the lighter the signaling cost and the larger the number of nodes that may be connected to the network. MVFS is much more efficient in terms of coupling efficiency than RSA. Let us consider the very simple case of a 2 nodes network. Figure 10 illustrates network state at the beginning of a given slot. With RSA, the probability to choose $E_{1}$ at the first step of the algorithm is 0.5 . The probability to choose $R_{2}$ at the second step is 0.5 . In that case, one may notice that vertex $E_{2}$ cannot be selected for the next transmission. In other words, the average coupling given by R.S.A is 1.75 . With MVFS, the first vertex to be selected is $R_{1}$. Then $E_{1}$ is selected. After having removed $E_{1}, R_{1}$ and the associated edge, $R_{2}$ and $E_{2}$ are 
always selected for the next transmission. As a conclusion, the MVFS allows for this simple example an average coupling of 2 instead of 1.75 with the RSA. We shall see in the following section, that this improvement is not negligible in terms of access delay for mederate to high loads.

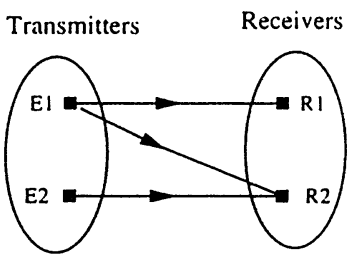

Figure 10: A comparison between the MVFS scheduling and the RSA scheduling.

\subsection{Performance analysis}

In all our simulations, stations are assumed equidistant. Propagation delay on the signaling channel is neglected. Each station $i$ generates packets during a slot according to a Bernoulli process. Let $p$ be the probability that station $i$ generates a packet during a slot. Let $p_{i, j}$ be the probability that station $i$ generates a packet for station $j$ during a slot:

$$
p=\sum_{j=1}^{N} p_{i, j} \leq 1
$$

One assumes in all our simulations that the $N$ stations of the network generate a same traffic load $\rho$. This offered load is thus given by probability $p$. In the following, $G$ stands for the number of groups. For each simulation result, the vertical axis corresponds to the mean packet access delay expressed in slot durations. The horizontal axis corresponds to the offered load $p$. Several access protocols are compared: TDM, pure RSA $(G=1)$, pure MVFS $(G=1)$, GTDM+RSA and GTDM+MVFS.

\subsubsection{Behaviour under symmetrical traffic}

Let us consider a passive optical star network with $N=8$ nodes. A packet generated by station $i$ has the same probability to be enqueued in any queue $j$, that is $p_{i, j}=$ $p / N, \forall j \in\{1,2, \ldots, N\}$. The groups are chosen of the same size. Mean packet access delay $E\left[d_{i, j}\right]$ for the TDM scheme has been derived in [7]:

$$
E\left[d_{i, j}\right]=1+\frac{(N-1)}{2\left(1-N \cdot p_{i, j}\right)}
$$

In the case of symmetrical traffic, one has: 


$$
E[d]=1+\frac{(N-1)}{2(1-p)}
$$

Figure 11 describes by means of a semi-logarithmic scale the evolution of mean packet access delay versus offered load. Under low load, the TDM scheme presents the highest access delays, around 4.5 slot durations, in coherence with the above analytical expression. Mean access delay $E[d]$ for pure RSA $(G=1)$ has been derived in [4] in the case of symmetrical traffic:

$$
E[d]=\frac{(1-p / N)}{(1-p)}
$$

As mentionned in [4], we confirm that this analytical result is very accurate for any load. For instance, under low load, $E[d]=1$, for a load of $0.8, E[d]=4.5$. The gap between the curve of TDM with the curves relative to dynamic allocation schemes increases with the offered load. Pure RSA and pure MVFS offers respectively better access delays than hybrid schemes like GTDM+RSA and GTDM+MVFS in the case of 2 groups $(G=2)$. One notices that over a $50 \%$ load, GTDM+MVFS offers lower access delays than GTDM+RSA. This improvement of about $50 \%$ under high loads. Larger number of groups are considered in Figure 12. For both GTDM+RSA and GTDM+MVFS, the larger $G$, the longer access delays. This result is quite intuitive. If the number of groups $G$ tends towards $N$, both GTDM+RSA and GTDM+MVFS behave like the TDM scheme. On the other hand, the larger $G$, the greater the gain in the signaling cost. The choice of $G$ is then the result of a compromise.

\section{Symmetrical traffic, $\mathrm{N}=8$}

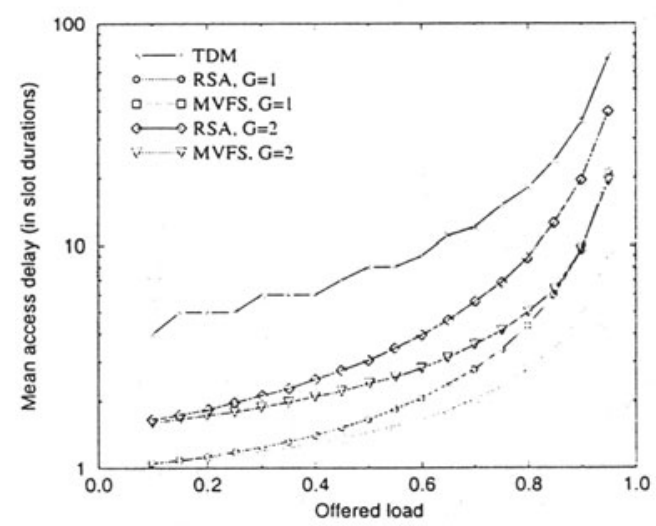

Figure 11: Mean access delay versus offered load, $G=1$ or $2, N=8$. 
An analytical expression of mean access delay has been derived in [6] for GTDM+RSA in the case of symmetrical traffic:

$$
E[d]=\frac{(1-p / N)}{(q-p / N)}+\frac{(G-1) q\left\{(2-q) p / N-(p / N)^{2}\right\}}{2 p / N(q-p / N)(q-G p / N)}
$$

If $k$ stands for the number of stations in a group $(G=N / k)$, the parameter $q$ is approximatively given by:

$$
q=\left\{1-\frac{(k-1) p}{k}\right\}
$$

\section{Symmetrical traffic, $N=8$}

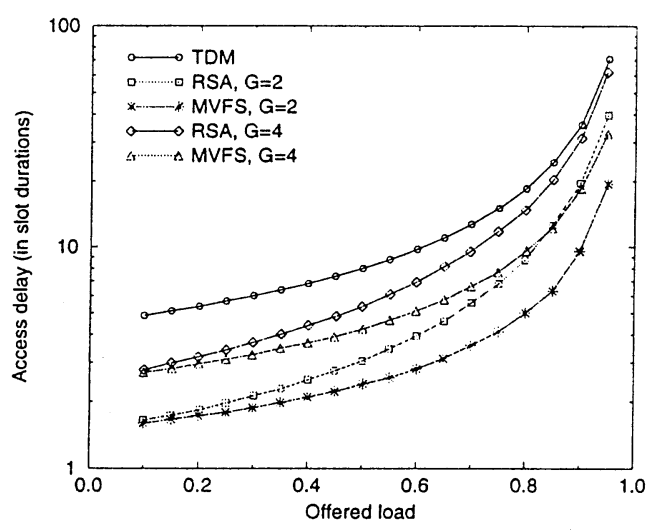

Figure 12: Mean access delay versus offered load, $G=2$ or $4, N=8$.

One notices that if $k=N$, the first term in the expression of $\mathrm{E}[d]$ corresponds to the average access delay of pure RSA. Under low load, Figure 12 confirms the above analytical expression of $E[d]$. Indeed, one has:

$$
\lim _{p \rightarrow 0} E[d]=1+\frac{(G-1)}{2}
$$

Thus, in the case of $G=2, \mathrm{E}[d]$ tends towards 1.5 , and in the case of $G=4, \mathrm{E}[d]$ tends towards 2.4. Figures 13 and 14 considers the case of a network with $N=20$ stations and respectively a number of groups $G=1$ or 2 (Figure 13 ), and $G=4$ or 10 (Figure 14).

Under low load, one notices that, as expected, the larger $N$, the longer access delays for TDM. Equation (8) shows that $\mathrm{E}[d]$ tends to $N / 2$ when $N$ is getting very large. We underline here the main drawback of TDM. Pure RSA, pure MVFS, GTDM+RSA and GTDM+MVFS keep roughly the same access delays under low loads when $N$ increases. 
Symmetrical traffic, $\mathrm{N}=20$

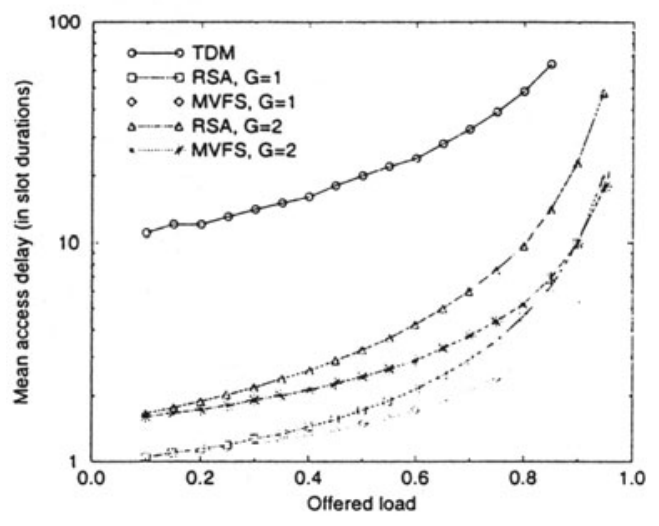

Figure 13: Mean access delay versus offered load, $G=1$ or $2, N=20$.

The GTDM+MVFS offers in any configuration the lowest access delays. The number of groups $G$ may be chosen according to the number of nodes connected to the network.

\subsubsection{Behaviour under asymmetrical traffic}

We consider now the case of an asymmetrical traffic on a passive optical star with $N=8$ nodes. Propagation delay is still neglected. The traffic matrix $P$ is such as :

$$
P=\left(\begin{array}{cccccccc}
0.01 & 0.01 & 0.01 & 0.01 & p & p & p & p \\
0.01 & 0.01 & 0.01 & 0.01 & p & p & p & p \\
0.01 & 0.01 & 0.01 & 0.01 & p & p & p & p \\
0.01 & 0.01 & 0.01 & 0.01 & p & p & p & p \\
0.1 & 0.1 & 0.1 & 0.1 & 0.01 & 0.01 & 0.01 & 0.01 \\
0.1 & 0.1 & 0.1 & 0.1 & 0.01 & 0.01 & 0.01 & 0.01 \\
0.1 & 0.1 & 0.1 & 0.1 & 0.01 & 0.01 & 0.01 & 0.01 \\
0.1 & 0.1 & 0.1 & 0.1 & 0.01 & 0.01 & 0.01 & 0.01
\end{array}\right)
$$

Each component $p_{i, j}$ of the matrix is such that $i$ stands for the transmitter address and $j$ stands dor the receiver address. The set of nodes $\{5,6,7,8\}$ generate a fixed traffic which is in big part intended for nodes $1,2,3$ and 4 . The set of nodes $\{1,2,3,4\}$ generate a fixed and low traffic intended for nodes belonging to the same set. This same set of nodes globally generate a variable traffic with intensity $4 p$ equally intended for nodes $5,6,7$ and 8 . In our simulations, $4 p$ fluctuates from 0 to 0.96 . In the case $G=2$, we have determined randomly which nodes belong to each group. In Figure 15, 


\section{Symmetrical traffic, $\mathrm{N}=20$}

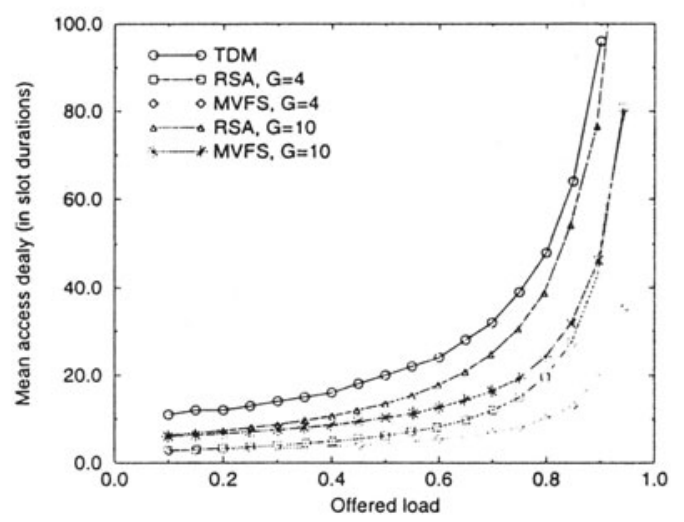

Figure 14: Mean access delay versus offered load, $G=4$ or $10, N=20$.

one has plotted mean access delays versus $p$ for the queue $j=5$ of node $i=1$. Using equation (7), we check the validity of TDM access delays under asymmetrical traffic. If $p_{1,5}=p=0.05, \mathrm{E}\left[d_{1,5}\right]=6.8$. When $p_{1,5}$ tends to 0.125 (that is $1 / 8$ ), $\mathrm{E}\left[d_{1,5}\right]$ tends to infinity. Again, pure MVFS gives the lowest access delays and GTDM+MVFS offers lower access delays than pure RSA for loads over 0.2 .

The best choice for the groups consists in separating the nodes generating a high load for the same destinations. One reduces like this the conflict probability. In the case of $G=4$ (which has not been simulated), the following groups should allow the best performance: $g_{1}=\{1,5\}, g_{2}=\{2,6\}, g_{3}=\{3,7\}, g_{4}=\{4,8\}$.

\section{Influence of propagation delays}

We underline in this section the disruptive effect that may result from propagation delay $\tau$ on the DAS-type protocols performance. Several papers assume that access delays with non-zero propagation time may be simply deducted from access delays with zero propagation time by adding the constant factor $\tau$. Let us consider the example described in Figure 16. This figure represents the evolution of the state of a buffer in a given node connected on a passive optical star network. One assumes a propagation delay $\tau$ of 4 slot durations. The observed station generates a packet every $2 \tau$. For the sake of simplicity, one assumes that the RSA always authorizes this station to dequeue a packet from the considered buffer. A first packet is generated at instant $t_{1}$. Simultaneously, the station sends an information in a minislot on the signaling channel to inform other stations. The RSA algorithm is activated only at instant $t_{5}$, just after 
Asymmetrical traffic, $\mathrm{N}=8$

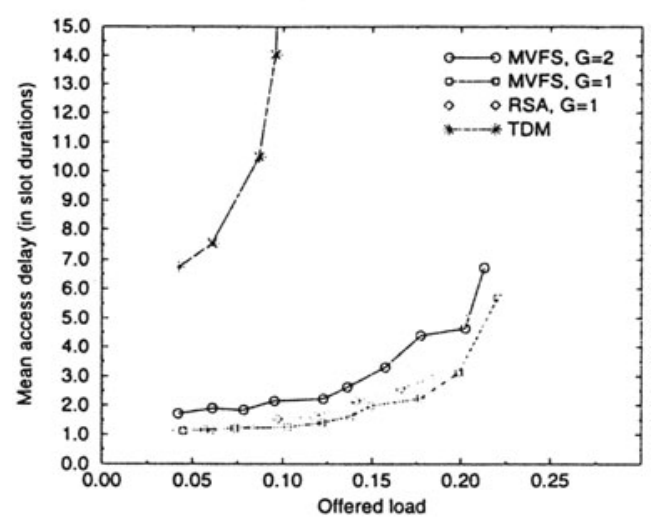

Figure 15: Mean access delay versus offered load, $G=1$ or $2, N=8$.

the minislot has been received by the other nodes of the network. We assume in our example that the RSA processing time is negligible. The observed station is allowed to transmit its packet at instant $t_{5}$. Several remarks may then be done:

- The packet generated at $t_{1}$ is transmitted 4 slots later at $t_{5}$.

- Because it cannot anticipate on the result of the RSA, the station continues to send a bit " 1 " on the same minislot of the signaling channel until instant $t_{5}$.

- Slots beginning at $t_{6}, t_{7}$ and $t_{8}$ are reserved by mistake.

- At instant $t_{9}$, a second packet is generated. This packet cannot benefit of the 3 "useless" previous signaling bits.

- If a packet had been generated at instant $t_{6}$ or $t_{7}$ or $t_{8}$, it should have benefit of a "useless" reserved slot. Its access delay should have been null, whereas the first packet access delay is 4 .

Thus, in the case of a periodic traffic where a packet is sent every $2 \tau$, the RSA reverves $50 \%$ of the bandwidth instead of one slot every $2 \tau$. The larger $\tau$, the least efficient the RSA. The parallel queueing does not suffer from the same inefficiency.

\section{Conclusion}

Passive optical star coupler networks are a very promising alternative in the field of Gigabit/s Local Area Networks. Optical packet switching requires dynamic wavelength 


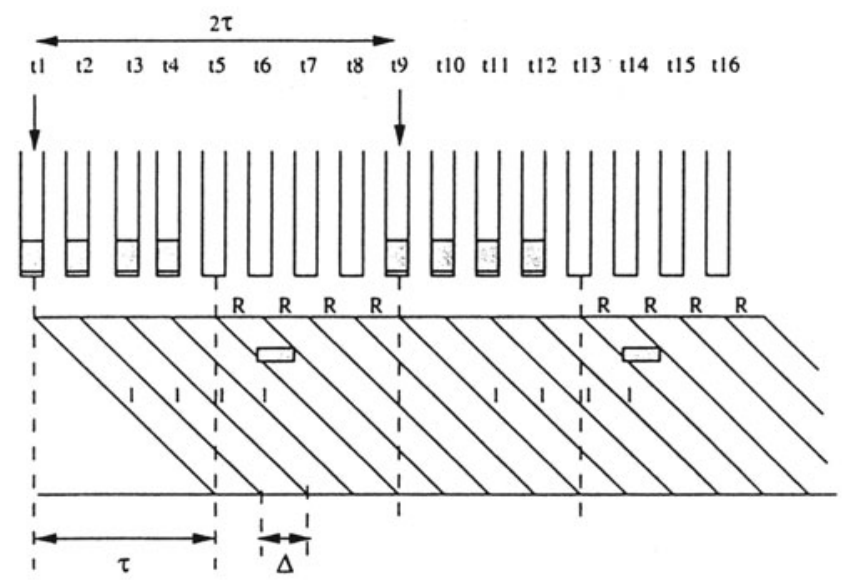

Figure 16: Behaviour anomaly of RSA scheduling due to propagation delay.

allocation schemes. Such schemes are sensitive to contentions. Conflict-free access is obtained by means of a signaling channel allowing each station to have a partial knowledge of the network load. In this paper, we have proposed a new MAC protocol which cumulates the benefits of the Group Time Division Multiplexing (GTDM) with those of the Minimum-degree Vertex First Scheduling (MVFS). GTDM allows a lightweight signaling. By this fact, a larger number of nodes may be connected to the network. We have underlined the fact that the MVFS offers a better coupling efficiency than the Random Scheduling Algorithm (RSA). The new proposed protocol (GTDM+MVFS) has been compared by computer simulations with other existing protocols such as TDM, pure RSA and GTDM+RSA. Pure MVFS allows the lowest access delays for moderate to high loads. In the case of symmetrical traffic, for loads greater than $50 \%$ and for a given number of groups, GTDM+MVFS offers better performance than GTDM+RSA. We have underlined the disruptive influence that propagation delays may have on DAS-type protocols. Under high loads, GTDM+MVFS offers better access delays and a lighter signaling cost than GTDM+RSA. The same conclusion has been obtainded in the case of asymmetrical traffic conditions. In a coming paper, we shall propose a new slot reservation mechanism for the GTDM+MVFS able to reduce this drawback.

\section{References}

[1] I. Cidon and Y. Ofeq, "Distributed Fairness for Local Area Networks with concurrent Transmission", Proceedings of the 3rd International Workshop on Distributed Algorithms, pp. 57-69, September 1989. 
[2] W. W. Lemppenau, H. R. Van As, H. R. Schindler, "A 2.4 Gbit/s ATM implementation of the CRMA-II dual ring LAN and MAN", Proceedings of EFOCN'93, The Hague, 1993.

[3] H. Kasahara, K. Imai, N. Morita, T. Ito, "Distributed ATM ring-based switching architecture for MAN and B-ISDN access networks", Proceedings of the first IFIP Conference on Broadband Communications, Estoril, 1992.

[4] R. Chipalkatti, A. S. Acampora, "Protocols for Optical Star-Coupler Network using WDM: Performace and complexity study", IEEE Journal on Selected Arra.s in. Communications, Vol. 11, No. 4, May 1994.

[5] D. Guo, Y. Yemini, Z. Zhang, "Scalable High-Speed Protocols for WDM Optical Star Networks", Proceedings of IEEE Infocom, Vol. 3, pp. 1544-1551, Toronto, June 14-16, 1994.

[6] B. Kannan, S. Fotedar, M. Gerla, "A protocol for WDM Star Coupler Networks", Proceedings of IEEE Infocom, Vol. 3, pp. 1536-1543, Toronto, June 14-16, 1994.

[7] I. Rubin, Z. Zhang, "Message Delay Analysis for TDMA schemes using contiguousslot assignments", IEEE Transactions on Communications, Vol. 40, pp. 730-737, April 1992. 